\title{
Die europäische Gewerkschaftsbewegung und die Gemeinsame Agrarpolitik (1958-1972): Annäherung an einen neuen Forschungsgegenstand
}

\author{
Rainer FATTMANN
}

\section{Voraussetzungen: \\ Die Gewerkschaften und das Projekt der europäischen Integration}

Schon die Anfänge der Europäischen Bewegung waren durch die aktive Mitarbeit zahlreicher Führungspersönlichkeiten der Freien wie auch der Christlichen Gewerkschaften der sechs Staaten gekennzeichnet, die später die Europäische Gemeinschaft für Kohle und Stahl (EGKS) und dann die Europäische Wirtschafts- sowie die Europäische Atomgemeinschaft bilden sollten. ${ }^{1}$ Viele ihrer Repräsentanten hatten bereits das European Recovery Program und im Zusammenhang damit die Vision einer wirtschaftlichen und politischen Einigung Europas in aller Regel uneingeschränkt, ja nicht selten mit Begeisterung begrüßt. Ein zunehmend integriertes Europa erschien ihnen nicht nur als Garant für einen dauerhaften Frieden in Europa und damit als notwendige Antwort auf die Verheerungen der beiden Weltkriege; die Einigung Europas galt darüber hinaus - jedenfalls in den Augen zentraler Führungspersönlichkeiten sowohl der sozialistisch-sozialdemokratisch geprägten, „freien“ Gewerkschaftsbewegung wie auch ihres christlich orientierten Pendants - als wichtige, wenn nicht unabdingbare Voraussetzung für den wirtschaftlichen Wiederaufbau Europas und das wirtschaftliche und soziale Wohlergehen der Arbeiterschaft der europäischen Staaten. ${ }^{2}$

Als 1955 Jean Monnet sein „Aktionskomitee für die Vereinigten Staaten von Europa“" aus der Taufe hob, zählten zu den 35 Gründungsmitgliedern des Komitees daher keineswegs zufällig nicht weniger als 14 Gewerkschaftsvertreter, die in ihren Heimatorganisationen ausschließlich hohe und höchste Posten bekleideten. Auch die Finanzierung dieses über jahrzehntelang überaus einflussreichen proeuropäischen

1. Der vorliegende Aufsatz präsentiert einige Ergebnisse eines von der Hans-Böckler-Stiftung geförderten Forschungsprojekts über die Geschichte der „Europäischen Föderation der Gewerkschaften des Lebensmittel-, Genussmittel-, Landwirtschafts- und Tourismussektors (EFFAT) und ihrer Vorläuferorganisationen“. Die Veröffentlichung des gesamten Forschungsberichts ist geplant.

2. Auskunft über die Hilfestellung der Gewerkschaftsbewegung auf dem Weg nach Europa finden sich an mehreren Stellen der Erinnerungen Jean Monnets; vgl. J. MONNET, Mémoires, Fayard, Paris, 1976. Den detailliertesten Überblick über die Rolle der Gewerkschaften als treibende Kräfte des europäischen Einigungsprozesses bietet nach wie vor E. HAAS, The Uniting Europe. Political, Social and Economic Forces 1950-1957, Stanford University Press, Stanford, 1958. 
,think tanks' wurde im Übrigen in erheblichem Maße von den Gewerkschaften, nicht zuletzt vom Deutschen Gewerkschaftsbund (DGB) geleistet. ${ }^{3}$

Vor diesem Hintergrund hatten die Spitzenfunktionäre der Christlichen wie diejenigen der Freien Gewerkschaften bereits den von Robert Schuman lancierten Plan einer supranationalen Montanunion nahezu einhellig begrüßt. Begünstigend für die gewerkschaftlichen Forderungen und ihre anhaltende Unterstützung dieses Projektes wirkte sich aus, dass in einer Zeit, in der der Ausgang des politischen und ökonomischen Konkurrenzkampfes zwischen Ost und West noch keineswegs entschieden war, seitens der westeuropäischen Regierungen, egal welcher Couleur, zunächst eine grundsätzlich hohe Bereitschaft bestand, die Arbeiterschaft und ihre (nichtkommunistischen) Repräsentanten in die Politik der europäischen Integration möglichst weitgehend einzubinden. ${ }^{4}$

Als sich die Außenminister der ,Sechs' auf der Konferenz von Messina (1. bis 3. Juni 1955) auf eine Resolution verständigten, in der die Grundzüge und die ersten Schritte zur Gründung einer Europäischen Wirtschaftsgemeinschaft (EWG) sowie einer Europäischen Atomgemeinschaft (EAG) proklamiert wurden, fand dieses Vorhaben einmal mehr die lebhafte Unterstützung der freien und christlichen Gewerkschaften der sechs beteiligten Nationen. Anders als noch während der Verhandlungen über die Einrichtung der Montanunion gelang es ihren Repräsentanten jedoch nur in geringem Umfang, den zu den Römischen Verträgen und damit zur Einrichtung der EWG und von Euratom führenden Aushandlungsprozess in ihrem Sinne zu beeinflussen. ${ }^{5}$

3. Darunter Robert Botherau und Georges Bouladoux, die Generalsekretäre der (sozialistisch orientierten) Confédération Générale du Travail - Force Ouvrière (FO) und der konkurrierenden christlichen Gewerkschaftsföderation, Confédération Française des Travailleurs Chrétiens (CFTC), August Cool, der über Jahrzehnte als der führende Repräsentant nicht allein der belgischen, sondern der internationalen Christlichen Gewerkschaftsbewegung anzusehen ist, André Renard, der Generalsekretär des freigewerkschaftlichen belgischen Gewerkschaftsbundes, Fédération Générale du Travail de Belgique (FGTB), sein Luxemburger Kollege Antoine Krier, Hendrik Oosterhuis, Präsident des (ebenfalls freigewerkschaftlichen) Nederlands Verbond van Vakverenigingen' (NVV), Guilio Pastore, der Generalsekretär der italienischen Confederazione Italiana Sindacati Lavoratori (CISL) und von deutscher Seite Walter Freitag, der Vorsitzende des Deutschen Gewerkschaftsbundes (DGB) und Heinrich Imig, derjenige der Bergarbeitergewerkschaft - um nur einige der wichtigsten zu nennen. Vgl. A. VARSORI, Jean Monnet et il Comitato d'Azione per gli Stati Uniti d'Europa dalla origini ai Trattati di Roma, in: A. CIAMPANI (Hrsg.), L' altra via per l'Europa. Forze sociali e organizzazione degli interessi nell'integrazione europea (1947-1957), Franco Angeli, Mailand, 1995, S. 139-170.

4. Vgl. E. HAAS, op.cit., insbes. S.214-239 und S.333-389; C. GOBIN, Consultation et concertation sociales à l'échelle de la Communauté économique: étude des positions et stratégies de la Confédération européenne des syndicats (1958-1991), 2 Bde., Diss., Brüssel, 1997, Bd.1, S.152-185.

5. Vgl. C. GOBIN, op.cit., Bd.1, S.200 f. Freie und Christliche Gewerkschaften forderten unisono, ana$\log \mathrm{zu}$ den Regelungen der Montanunion, dass sich unter den Mitgliedern der zu schaffenden Kommissionen ein Vertreter der Gewerkschaftsbewegung befinden müsse. Zugleich sollten die zu gründenden Gemeinschaften wie die EGKS mit sozialpolitischen Kompetenzen ausgestattet und, wieder nach dem Vorbild der Luxemburger Organisation, mit eigenen Finanzmitteln ausgestattet werden. Gefordert wurde von Vornherein auch die Harmonisierung der sozialpolitischen Regelwerke der Mitgliedsstaaten „nach oben“, ein Ansinnen, das über Jahrzehnte immer wieder formuliert wurde und 
Obwohl die konkreten Forderungen der Freien und Christlichen Gewerkschaften der Sechs in Bezug auf die Ausgestaltung insbesondere der EWG schließlich allenfalls in nur sehr geringem Umfang erfüllt wurden, begrüßten sie nach wie vor trotz aller Vorbehalte und Kritik die Gründung von EWG und EAG als ,relance européene“, als Neubeginn weiterer europäischer Integrationsschritte. Nicht nur Robert Bothereau, der Generalsekretär des freigewerkschaftlichen französischen Gewerkschaftsbundes Confédération Générale du Travail - Force Ouvrière (FO), sah mit der Unterzeichnung der Römischen Verträge die „Stunde Europas“ gekommen. ${ }^{6}$

\section{Gewerkschaftliche Interessenvertretung innerhalb der EWG}

Am 16. Januar 1958 - und damit am gleichen Tag an dem die EWG-Kommission ihre Tätigkeit aufnahm - fand in Düsseldorf die konstituierende „Generalversammlung der freien Gewerkschaften der Mitgliedstaaten der Europäischen Gemeinschaft (IBFG)" statt. Während dieser Sitzung wurde die Errichtung eines allein auf die nunmehr drei Europäischen Gemeinschaften bezogenen „Europäischen Gewerkschaftssekretariats" (EGS) beschlossen und ein Exekutivausschuss gewählt, der, zusammen mit dem Sekretariat, die operative Politik der Freien Gewerkschaften in den folgenden Jahren entscheidend prägen sollte. ${ }^{7}$

In den folgenden Jahren sollte sich das EGS ${ }^{8}$ als Schnittstelle und zentraler Akteur (frei-) gewerkschaftlicher Politik gegenüber den europäischen Institutionen etablieren, und zwar einerseits branchenübergreifend, aber auch in denjenigen Sektoren, die entsprechend des EWG-Vertrages in den „unmittelbaren Zuständigkeitsbereich“ der neu gegründeten Gemeinschaft fielen und damit insbesondere im Landwirtschaftsbereich. ${ }^{9}$ Am 7. Mai 1958 entschlossen sich die Christlichen Gewerkschaften der Sechs eine eigenständige Gewerkschaftsformation auf europäischer Ebene zu bilden,

immer wieder auf harten Widerstand der Mehrzahl der verantwortlichen Regierungen stieß. Des Weiteren sollte, dies war von Beginn an eine Schlüsselvorstellung der europäischen Arbeitnehmerorganisationen, ein Wirtschafts- und Sozialausschuss eingerichtet werden, der mindestens über dieselben Befugnisse verfügen sollte wie der Beratende Ausschuss der EGKS. Zwar gelang schließlich auf gewerkschaftlichen Druck und insbesondere Dank der belgischen Delegation die Einrichtung eines solchen Ausschusses, seine Kompetenzen blieben jedoch hinter denjenigen seines Luxemburger Pendants und damit hinter den Forderungen der Arbeitnehmervertreter weit zurück.

6. R. BOTHEREAU, L'heure européenne, in: Force ouvrière, 24.01.1957, S.1.

7. Vgl. C. GOBIN,, op.cit., Bd.1, S.204-359.

8. Im eigentlichen Sinne war das EGS das administrative Organ der „Freien Gewerkschaften innerhalb der Europäischen Gemeinschaften (IBFG)"; allerdings hat es sich eingebürgert, auch diese Gewerkschaftsföderation selbst als EGS zu bezeichnen (engl.: ETUS; franz.: SSE).

9. In den „unmittelbaren Zuständigkeitsbereich“ der Gemeinschaften fiel neben dem Agrarsektor das Transportwesen sowie - im Rahmen von EURATOM - die Nuklearwirtschaft. 
die Europäische Organisation des Internationalen Bundes der Christlichen-Gewerkschaften (OE-CISL). ${ }^{10}$

Dabei ist die in weiten Teilen der Literatur vorherrschende Einschätzung des EGS, also des Zusammenschlusses der sechs IBFG-Gewerkschaftsdachverbände, als schwach und wenig handlungsfähig, außerordentlich problematisch, ja, als unzutreffend anzusehen. ${ }^{11}$ Trotz des bis zur Umwandlung des EGS in den Europäischen Bund der Freien Gewerkschaften (EBFG) im Jahr 1967 praktizierten Einstimmigkeitsprinzips scheint das EGS zu allen wichtigen politischen Fragen, wohl in erster Linie auf Grund der gewerkschaftsübergreifenden proeuropäischen Grundorientierung, zu dezidierten und klaren Stellungnahmen gefunden zu haben. ${ }^{12}$ Dabei hatten sowohl die christlich wie auch die sozialistisch orientierten Gewerkschaften der sechs Mitgliedsstaaten in allen relevanten europapolitischen Fragen bereits in den 1950er Jahren an einem Strang gezogen. Seit den frühen 1960er Jahren stimmten die Generalsekretäre des EGS und der OE-CISC - Harm G. Buiter und Jan Kulakowski - die europapolitischen Forderungen und Initiativen ihrer Organisationen immer enger aufeinander $a b$, so dass die nichtkommunistischen Gewerkschaftsföderationen gegenüber den europäischen Institutionen mit einer Stimme zu sprechen in der Lage waren. Von der in weiten Teilen der Literatur beschworenen vermeintlichen ,ideologische Zerrissenheit" der europäischen Gewerkschaftsbewegung ${ }^{13}$ in den späten 1950er und 1960er Jahren - sieht man von den kommunistischen Organisationen einmal ab kann somit hinsichtlich ihrer Europapolitik keine Rede sein. ${ }^{14}$

10. Organisation européenne de la CISC. Kern der Gruppe waren die christlichen Gewerkschaften Belgiens (CIC), der Niederlande (NKV und CNV) und die französische Confédération française des travailleurs chrétiens (CFTC). Vgl. P. PASTURE, Histoire du syndicalisme chrétien international. La difficile recherche d'une troisième voie, L'Harmattan, Paris, 1999, S.266-286.

11. Zum Forschungsstand (in Deutschland) vgl. S. REMEKE, Gewerkschaften als Motoren der europäischen Integration: Der DGB und das soziale Europa von den Römischen Verträgen bis zu den Pariser Gipfelkonferenzen (1957-1974), in: Mitteilungsblatt des Instituts für soziale Bewegungen, 2009, S.63-86.

12. Das betraf übergeordnete europapolitische Probleme wie die Frage der Erweiterung der Gemeinschaften, die ablehnende Haltung gegenüber der Obstruktionspolitik de Gaulles 1965/66, die Forderung nach erweiterten Rechten der europäischen Institutionen, insbesondere des Wirtschafts- und Sozialausschusses (WSA) und des Europäischen Parlamentes, dessen Direktwahl immer wieder gefordert wurde, aber auch konkrete gewerkschaftspolitische Forderungen, wie diejenige nach einer gemeinschaftlichen europäischen Sozialpolitik und einer Angleichung der sozialen Standards im Europa der Sechs ,nach oben'.

13. Dieses gerade in der deutschsprachigen Gewerkschaftsgeschichtsschreibung seit Jahrzehnten wiederholte (Fehl-)Urteil findet sich zuletzt noch in der Übersichtsdarstellung über die Bestände der europäischen Gewerkschaftsverbände, die im Archiv der sozialen Demokratie der Friedrich Ebert Stiftung in Bonn verwahrt werden; vgl. W. BUSCHAK, Der Europäische Gewerkschaftsbund und die Europäischen Gewerkschaftsverbände, in: Europäische Gewerkschaftsorganisationen. Bestände Im Archiv der Sozialen Demokratie und in der Bibliothek der Friedrich-Ebert-Stiftung, 2. erw. Auflage, Dietz, Bonn, 2007, S.9-19.

14. Vgl. C.R. BEEVER, European Unity and the Trade Union Movement, Sythoff, Leiden, 1960; P. PASTURE, Trade unions as a transnational movement in the European space 1955-1965. Falling short of ambitions?, in: W. KAISER , P. STARIE (Hrsg.), Transnational European Union, Routledge, Milton Park, 2006, S.109-130. Demgegenüber scheint sich der 1973 gegründete Europäische 


\section{Die Herausbildung der Gemeinsamen Agrarpolitik und die Gründung der „Europäischen Föderation der Agrargewerkschaften“}

Die Unterzeichnung der Römischen Verträge am 25. März 1957 stellte nicht zuletzt die Agrargewerkschaften der sechs Mitgliedsstaaten vor neue Herausforderungen: Artikel 3 des EWG-Vertrages erklärte die Gemeinsame Agrarpolitik zu einem der primären Tätigkeits- und Zuständigkeitsfelder der Gemeinschaft; Artikel 43 EWGV bestimmte, dass die als Exekutivorgan der EWG gegründete Europäische Kommission unmittelbar nach Inkrafttreten des Vertrags eine Konferenz der Mitgliedsstaaten einzuberufen habe, die die Leitlinien der künftigen Gemeinsamen Agrarpolitik (GAP) ausarbeiten sollte. Diese Landwirtschaftskonferenz fand vom 3. bis 12. Juli 1958 im italienischen Badeort Stresa am Lago Maggiore statt. ${ }^{15}$

Schon am Rande der 14. Konferenz der Internationalen Landarbeiter-Föderation (der schon 1920 gegründeten Gewerkschaftsinternationale der Agrargewerkschaften), die am 6. und 7. Juni 1957 in Genf getagt hatte, hatten sich die Vertreter der Landarbeitergewerkschaften der künftigen EWG-Staaten darauf verständigt, in naher Zukunft zu einer Diskussion der anstehenden Fragen und Probleme zusammen zu kommen. Auf Einladung der niederländischen Landarbeitergewerkschaft trafen sich Repräsentanten der freien Agrargewerkschaften in der EWG erstmals am 11. und 12. März 1958 zu einer gemeinsamen Konferenz in Luxemburg. Anwesend waren Vertreter von acht Gründungsorganisationen aus fünf EWG-Staaten. ${ }^{16}$ Die Luxemburger Konferenz kann als Gründungskonferenz der späteren „Europäischen Föderation der Agrargewerkschaften“ (EFA) angesehen werden. Denn mit der „Arbeitsgruppe Europäischer Landarbeitergewerkschaften“" wurde nunmehr ein gewerkschaftlicher Zusammenschluss der Landarbeitergewerkschaften auf EWG-Ebene aus der Taufe gehoben. Auffallend war von Beginn an, dass ihre Mitgliederstruktur nicht nur Landarbeitergewerkschaften umfasste, sondern auch zwei italienische Verbände,

Gewerkschaftsbund (EGB), trotz des von seinen Gremien nunmehr endgültig praktizierten Mehrheitsprinzips, über Jahre hinweg in einem Zustand der Selbstlähmung befunden zu haben. Dies war offenbar eine Folge seiner zunächst nur geringfügigen programmatischen und politischen Kohäsion infolge des nunmehr fehlenden proeuropäischen Grundkonsenses der beteiligten Gewerkschaftsverbände nach dem Beitritt des Vereinigten Königreichs, Irlands und Dänemarks zur EG.

15. Vgl. A.-C.L. KNUDSEN, Farmers on Welfare. The Making of Europe's Common Agricultural Policy, Cornell University Press, Ithaca/London, 2009, S.123-130.

16. Vertreten waren: Centrale Générale, Belgien, Gewerkschaft Gartenbau, Land- und Forstwirtschaft (GGLF), Bundesrepublik Deutschland; Fédération Nationale Force Ouvrière de l'Agriculture et Secteurs Connexes (Frankreich); Federazione Italiana Coloni Mezzadri e Coltivatori Diretti (FEDERCOLTIVATRI - CISL), Federazione Italiana Salariati Braccianti Agricoli e Maestranze Specializzate (FISBA), Unione Italiana Mezzadri e Coltivatori Diretti (UIMEC), Unione Italiana Salariati Braccianti Agricoli (UISBA), alle vier: Italien; sowie der Agrarische en Voedingsbedrijfsbond (AVB), Niederlande (in Luxemburg existierte keine Agrargewerkschaft). 
die die Interessen der Kleinbauern und Pächter des Landes vertraten. ${ }^{17}$ Zum Präsidenten der Arbeitsgruppe wurde Hellmut Schmalz gewählt, der zu diesem Zeitpunkt stellvertretende Vorsitzende der deutschen „Gewerkschaft Gartenbau, Land- und Forstwirtschaft" (GGLF). Dass die EWG-Institutionen der neugegründeten Organisation durchaus Gewicht beimaßen, verdeutlichte die Anwesenheit des EWG-Agrarkommissars und stellvertretenden Kommissionspräsidenten Sicco Mansholt, der die Agrarpolitik der Gemeinschaft auf Jahrzehnte hinaus prägen sollte. ${ }^{18}$

Bis 1963 wurde die operative gewerkschaftspolitische Arbeit der EFA weitgehend vom EGS und ihrem Generalsekretär sowie dem Exekutivausschuss der freien Gewerkschaften geleistet, dem der EFA-Vorsitzende bereits bei dessen Konstitution im Januar 1958 angehört hatte. ${ }^{19} 1963$ wurde dann für den Agrarbereich mit Astrid Lulling erstmals eine Gewerkschaftssekretärin eingestellt, die allerdings zugleich den Lebensmittel- und Gastgewerbesektor betreute. Dass eine solche Position mit einer Frau besetzt wurde, war in den frühen 1960er Jahren nicht nur auf europäischer Gewerkschaftsebene höchst außergewöhnlich. ${ }^{20}$

17. Vgl. R. FATTMANN, Gewerkschaften ohne Grenzen - Für ein soziales Europa. 50 Jahre europäische Gewerkschaftspolitik im Agrar-, Lebensmittel- und Tourismusbereich 1958-2008, Friedrich-Ebert-Stiftung, Bonn, 2008, S.11. Gerade die italienische Landwirtschaft war noch weit über die 1960er Jahre hinaus durch zahlreiche zwischen Selbständigkeit und Lohnabhängigkeit changierende Beschäftigungsverhältnisse geprägt. Vgl. P. GINSBORG, A History of Contemporary Italy. Society and Politics 1943-1988, Palgrave Macmillan, London, 1990, S.106-110, S.121-140.

18. Mansholts Rolle innerhalb der Gemeinsamen Europäischen Agrarpolitik von den späten 1950er bis frühen 1970er Jahre kann kaum überschätzt werden. Zwischen 1945 und 1958 hatte er das Amt des niederländischen Landwirtschaftsministers innegehabt. Als (undogmatischer) Sozialist war der Agrarkommissar immer bereit, die Auffassungen der Gewerkschaften in seine Überlegungen mit einzubeziehen und die anstehenden Probleme im Agrarbereich mit ihnen zu diskutieren. Dabei legte er, dies belegen zahlreiche Zeugnisse, ein hohes Maß an Überzeugungskraft an den Tag, das selbst politische Gegner nicht unbeeindruckt ließ; zu Mansholt vgl. J.v. MERRIËNBOER, Mansholt. Een biografie, Boom, Amsterdam, 2006.

19. Bis Mitte der 1970er Jahre verfügte die EFA über keine eigene Finanzverwaltung. Die laufenden Ausgaben, insbesondere die Personalkosten des Sekretariats, wurden aus dem EGS- bzw. EGB Haushalt bestritten, in den die EFA-Verbände wiederum einen monatlichen Fixbetrag einzahlten. Vgl. hierzu die entsprechenden Protokolle über die Kongresse der EFA und die Tätigkeitsberichte ihres Sekretariats, die im EFA-Bestand des Archivs der sozialen Demokratie in der Friedrich-EbertStiftung (AdsD) vorliegen.

20. Der Grund hierfür lag offenkundig in der ebenso außergewöhnlichen Vita der Kandidatin: Die 1929 im luxemburgischen Schifflange geborene Lulling konnte trotz ihres jugendlichen Alters bereits über einen beeindruckenden Fundus an Erfahrungen in der europäischen Gewerkschaftsarbeit zurückgreifen: Neben ihrer Tätigkeit als Sekretärin und Redakteurin beim Lëtzebuerger ArbechterVerband (1949-1963) hatte sie zwischen 1950 und 1958 zugleich im Verbindungsbüro der Bergarbeiter und Metallarbeiter bei der EGKS in Luxemburg mitgearbeitet und die Herausbildung des europäischen Institutionengefüges von Beginn an wie nur wenige andere miterlebt. Sie führte das gemeinsame Sekretariat beider Organisationen bis zum Ende des Jahres 1971. In diesem Zeitraum war sie auch Vorsitzende der sozialistischen Frauenvereinigung der Lëtzebuerger Sozialistesch Arbechterpartei. Zudem wurde sie 1965 auf Vorschlag ihrer Partei in das Europäische Parlament gewählt, ein Mandat, von dem sie in ihrer Funktion gewiss profitierte und das andererseits beiden Gewerkschaftsföderationen einen unmittelbaren Zugang zu dieser - zu diesem Zeitpunkt allerdings noch nicht mit sehr weit reichenden Befugnissen ausgestatteten - europäischen Institution ermöglichte. 


\section{Das Verhältnis der EFA zu den europäischen Institutionen}

An der Herausbildung europäischer Gewerkschaftsstrukturen in der EWG hatte die Kommission einen bedeutenden Anteil, gerade auch im landwirtschaftlichen Bereich. Gegenüber den Agrargewerkschaften hatte Mansholt von Beginn an verdeutlicht, dass er mit Repräsentanten nationaler Verbände nicht zu verhandeln bereit sei und ein abgestimmtes Vorgehen und eine einheitliche Position der Gewerkschaften der Sechs verlangt. Letztere wurden so bis zu einem gewissen Grad zur Kooperation gezwungen. Bereits auf der Konferenz von Stresa war ein Vertreter der „Arbeitsgemeinschaft" vertreten. Mansholt nahm in den 1960er Jahren an sämtlichen EFAKongressen teil, ebenso wie der langjährige Sozialkommissar Lionelli Levi Sandri und häufig auch die zuständigen Generaldirektoren (GD V und VI). Während seiner Amtszeit stand Mansholt und die Generaldirektion VI - hier pflegte Georges Rencki die Kontakte zu den Berufsorganisationen (organisations professionelles) und damit auch zu den Gewerkschaften - in ständigem Kontakt und Dialog mit den zentralen Akteuren der EFA. Sowohl die EFA als auch das EGS bescheinigten dem Agrarkommissar immer wieder seine Bereitschaft zur Kooperation. Aber auch Walter Hallstein pflegte zeit seiner Präsidentschaft regelmäßige Kontakte zum EGS, auf dessen Generalversammlungen er den „Gefährten der ersten Stunde“ - so seine Ansprache an die gewerkschaftlichen Delegierten der 3. Generalversammlung der freien Gewerkschaften der Sechs - regelmäßig die Positionen und politischen Vorhaben seiner Kommission erläuterte. ${ }^{21}$

Zweifellos war die Kommission in den 1960er Jahren der wichtigste ,access point" der Gewerkschaften überhaupt und der EFA im Besonderen, der es ihnen ermöglichte, ihre Forderungen in die politischen Entscheidungsprozesse der EWG einzuspeisen. Die Repräsentanten der Gewerkschaften und die zentralen Mitglieder der ersten Kommissionen, insbesondere Hallstein und Mansholt, aber auch Persönlichkeiten wie Jean Rey, Robert Marjolin, Hans von der Groeben und Émile Noël, der über Jahrzehnte das Amt des Generalsekretärs der seit 1967 vereinigten Kommission der Gemeinschaften bekleidete, teilten, wenn auch gewiss in einigen Abschattierungen, das Ziel eines wirtschaftlich und politisch vereinten, mit supranationalen Institutionen ausgestatteten und zunehmend handlungsfähigen Europas. Und Gewerkschaften wie Kommission teilten, obwohl keinesfalls alle Mitglieder der Kommissionen der 1960er Jahre dem sozialistischen Parteienspektrum zuzuordnen waren, zumindest im Grundsatz den Grundgedanken, dass der zu schaffende Gemeinsame Markt einer gewissen Regulierung, Planung und gemeinschaftlicher Rahmensetzung bedürfe, nicht allein im Bereich der Landwirtschaft. ${ }^{22}$

21. Die entsprechenden Protokolle finden sich im EGB-Archiv des Internationalen Instituts für Sozialgeschichte Amsterdam (IISG).

22. Dies traf wohl selbst auf Hans von der Groeben zu, der bis 1970 als für den Wettbewerb zuständiges Kommissionsmitglied amtierte und der innerhalb der Riege der Kommissare, vielleicht neben Jean Rey, stets die liberalste und marktwirtschaftlichste Position einnahm. Allerdings gelangte auch er im Verlauf der 1960er Jahre zu der Auffassung, dass die Einrichtung des Gemeinsamen Marktes insbesondere durch eine gemeinschaftliche Struktur- und Regionalpolitik flankiert werden müsse - 
Insgesamt entsprach die Arbeit der Kommission in den ersten Jahren nach Gründung der EWG nicht nur den Vorstellungen der Gewerkschaften, sondern selbst denjenigen der sozialistischen bzw. sozialdemokratischen Parteien nicht nur in den Grundlinien, sondern häufig bis in die Details. Insbesondere ihr im Oktober 1962 vorgelegtes Aktionsprogramm für die zweite Stufe der wirtschaftlichen Integration in der sogenannten Übergangszeit (bis zur Herausbildung eines gemeinsamen Marktes), fand die ungeteilte Zustimmung sowohl der christlichen wie auch der freien Gewerkschaften und selbst der den letzteren eng verbundenen sozialistischen und sozialdemokratischen Parteien. ${ }^{23}$

Vor diesem Hintergrund unterstützten die nichtkommunistischen Gewerkschaften der Sechs die Kommission bis in die 1970er Jahre (und im Grundsatz sicherlich auch darüber hinaus) in allen Punkten von politischer Bedeutung und stärkten ihr auch in der Öffentlichkeit den Rücken. ${ }^{24}$ Auch die Agrargewerkschaften stellten sich stets hinter sie, beispielsweise im Jahr 1965 durch einen Aufruf an die französische Regierung, ihre Politik des ,leeren Stuhles' zu beenden und zur Mitarbeit innerhalb der europäischen Institutionen zurückzukehren. ${ }^{25}$

Im Gegenzug legte die Kommission, und namentlich Mansholt, von Beginn an großen Wert darauf, die Arbeitnehmervertreter in die europäischen Institutionen einzubinden. Schon 1961 organisierte die Kommission eine erste Konferenz der europäischen Sozialpartner, der EFA auf der einen Seite sowie der ebenfalls schon 1958 aus der Taufe gehobenen Dachorganisation der Bauernverbände, der COPA (Comité des Organisations Professionnelles Agricoles) ${ }^{26}$ auf der anderen, die vom 28. September bis zum 4. Oktober desselben Jahres in Rom stattfand und die nicht weniger als den Beginn des sozialen Dialogs in der EWG markierte. ${ }^{27}$ Zugleich band die Kommission die Vertreter der EFA und der ihr angeschlossenen nationalen Mitgliedsverbände in das sich immer weiter ausdehnende Geflecht beratender Ausschüsse ein, die die Kommission bei der Ausarbeitung der seit 1962 nach und nach auf den Weg ge-

gerade auch im Bereich der europäischen Agrarpolitik. Vgl. den Beitrag von Katja Seidel in diesem Heft und H.v.d. GROEBEN, Deutschland und Europa in einem unruhigen Jahrhundert. Erlebnisse und Betrachtungen, Nomos, Baden-Baden, 1995, S.356-362.

23. Vgl. I. ELSNER, Bericht der Arbeitsgemeinschaft - Die EWG als wirtschafts- und sozialpolitische Union, in: SPD-Parteivorstand (Hrsg.), Sorge um Europa: Europa-Kongress vom 25. bis 26. Februar 1964 in Bad Godesberg, Bonn, o-J. [1964], S.11-16.

24. Vgl. DGB, Bundesvorstand (Hrsg.), Vereinigte Staaten von Europa - Partner der freien Welt (Großkundgebung der Freien Gewerkschaften der EWG-Länder am 6. Juli 1963 in Dortmund), Düsseldorf, o-J. [1963].

25. AdsD, EFAA00003, Entschließung der Dritten Konferenz der Landarbeitergewerkschaften (IBFG) in der EWG am 18. und 19. September 1965 in Bari.

26. Zur Rolle der COPA vgl. J. MEYNAUD, D. SIDJANSKI, Les groupes de pression dans la Communaute européenne, Éd. de l'Institut de Sociologie, Brüssel, 1971, S.164-234.

27. Vgl. R. FENNELL, The Common Agricultural Policy, Continuity and Change, Oxford University Press, Oxford, 1997, S.32-35. 
brachten Marktordnungen für die verschiedenen landwirtschaftlichen Produkte beriet. $^{28}$

Obwohl dem Europäischen Parlament (EP) wie auch dem Wirtschafts- und Sozialausschuss (WSA) zumindest in den ersten beiden Jahrzehnten des europäischen Integrationsprozesses seitens der wissenschaftlichen Literatur nicht viel mehr als eine mehr oder minder dekorative Funktion im institutionellen Gefüge der Gemeinschaft zugebilligt werden, erwies sich die Zusammenarbeit der EFA mit beiden Gemeinschaftsorganen als von nicht zu unterschätzender Bedeutung. Namentlich die Hallstein-Kommission hatte den Stellungnahmen des WSA und insbesondere des Europäischen Parlaments stets einen hohen Stellenwert eingeräumt. Das lag zunächst daran, dass die Kommission die Versammlung als die legitime Repräsentation der Bevölkerung der Sechs erachtete und sich immer wieder für eine Erweiterung der Rechte des Parlaments stark gemacht hatte; eine Missachtung ihrer Vorschläge hätte diese Politik fraglos diskreditiert.

Bereits unmittelbar nach Gründung der EWG war es den Agrargewerkschaften dabei auch mit Hilfe ihnen verbundener Abgeordneter innerhalb des EP gelungen, die Kommission zu einer umfassenden Untersuchung über die soziale und wirtschaftliche Lage in den sechs Ländern der Gemeinschaft zu veranlassen..$^{29}$ Insgesamt verstanden es die Agrargewerkschaften, ihre Kanäle in das Europäische Parlament zu nutzen, um die für ihre Arbeit unverzichtbaren Informationen über die verschiedensten Aspekte der europäischen Agrarpolitik zu erlangen. Dabei verfügten sie mit Hendrikus (Henk) Vredeling innerhalb des Europäischen Parlaments seit Gründung der EWG über einen zentralen Ansprechpartner, der bereits auf der ersten Zusammenkunft der europäischen Agrargewerkschaften das Grundsatzreferat über die Situation der Landwirtschaft innerhalb der EWG gehalten hatte und der mit der Aufrechterhaltung der Kontakte in die Parlamentarische Versammlung betraut worden war. Vredeling, der als ehemaliger Gewerkschaftssekretär der niederländischen Landarbeitergewerkschaften über profunde Fachkenntnisse in allen Aspekten der Agrarpolitik verfügte, entwickelte sich in kürzester Zeit zum führenden Agrarexperten des Europäischen Parlaments. ${ }^{30}$ Als Berichterstatter des landwirtschaftlichen

28. Vgl. I. STÖCKL, Gewerkschaftsausschüsse in der EG. Die Entwicklung der transnationalen Organisation und Strategie der europäischen Fachgewerkschaften und ihre Möglichkeiten zur gewerkschaftlichen Interessenvertretung im Rahmen der Europäischen Gemeinschaft, Engel-Verlag, Keh1/Straßburg, 1986, S.62.

29. Vgl. Kommission der EWG (Hrsg.), Überblick über die Lage der landwirtschaftlichen Arbeitnehmer, Brüssel, 1960.

30. Vredeling, am 24.11.1924 im holländischen Amersfoort geboren, hatte seine berufliche Laufbahn Anfang der 1950er Jahre als Gewerkschaftssekretär der niederländischen Agrargewerkschaft (ANAP) begonnen und war 1956 für die PvdA in die Zweite Kammer der Generalstaaten gewählt worden. Von 1958 bis 1973 gehörte er zugleich dem Europäischen Parlament an. Von 1973 bis 1977 bekleidete er in seinem Heimatland das Amt des Verteidigungsministers, von 1977 bis 1981 war er unter Roy Jenkins Vizepräsident der Kommission und zuständig für den Bereich Arbeit und Soziales. Vredeling zählte wie neben ihm wohl nur Alfred Mozer zu den wirklich führenden Akteuren und bestens vernetzten Akteuren der europapolitischen Akteure des sozialistischen Parteienspektrums und sollte wie dieser in den 1970er Jahren bei der Herausbildung europäischer 
Ausschusses legte er bereits 1959 einen ersten Bericht über die Strukturprobleme der Landwirtschaft innerhalb der EWG vor, ${ }^{31}$ dem zahlreiche Stellungnahmen und Entschließungen des Parlaments zur Fortentwicklung der Gemeinsamen Agrarpolitik folgen sollten. Dabei war Vredeling nur einer von mehreren den Agrargewerkschaften nahestehenden oder auch angehörenden Akteuren in den europäischen Institutionen. Vor diesem Hintergrund verwundert es nicht, dass die seitens der europäischen Agrargewerkschaften entwickelten Konzepte zur Ausgestaltung der Gemeinsamen Agrarpolitik von Beginn an in enger Kooperation mit zentralen Akteuren sowohl innerhalb des EP wie des WSA erarbeitet worden sind. In diesem Rahmen wurde offenbar auch von Beginn an eine enge Kooperation mit den christlichen Agrargewerkschaften sicher gestellt. Über die Zusammenarbeit mit dem WSA in den ersten beiden Jahren nach Gründung der EWG berichtete Buiter für das EGS den Delegierten der zweiten Konferenz der Landarbeitergewerkschaften der Sechs:

„La prise de position au sujet des principales questions de la politique agricole, élaborée par notre groupe de travail avec quelques membres du Comité économique et social, parmi lesquels se trouvaient également des représentants des syndicats chrétiens, mérite d'être particulièrement souligné“. 32

\section{Die Beratenden Ausschüsse und der soziale Dialog in der Landwirtschaft}

Die gewerkschaftliche Mitwirkung im System der ,Beratenden Ausschüsse' gewann umso mehr an Gewicht, je mehr „Agrareuropa“ in den 1960er Jahren Gestalt gewann; sie ermöglichte es - jedenfalls bis zu einem gewissen Grade - auf die Entscheidungsfindungsprozesse in der Gemeinschaft Einfluss zu nehmen. Bis zum Ende der 1970er Jahre wurden allein im Bereich der Landwirtschaft nicht weniger als 27 solcher Ausschüsse geschaffen, die sich mit zum Teil sehr spezifischen Fragestellungen befassten. So existierte etwa ein „Ausschuss Eier“ und ein „Ausschuss Hopfen“. Beschickt wurden (und werden) diese Ausschüsse einerseits mit Vertretern der Bauern und der Genossenschaften, der Industrie und des Handels, andererseits der Arbeitnehmer und der Verbraucher. Hierbei agierten die europäischen Gewerkschaften bis weit in die 1970er Jahre, angesichts der zunächst nur schwachen Präsenz der Verbraucherverbände auf europäischer Ebene ${ }^{33}$ in der Praxis nicht zuletzt als Vertreter

Strukturen der europäischen Arbeiterparteien eine Schlüsselrolle spielen. Vgl. (A.v. GEHLEN, Europäische Parteiendemokratie? Institutionelle Voraussetzungen und Funktionsbedingungen der europäischen Parteien zur Minderung des Legitimationsdefizits der EU, Dissertation, Freie Univ. Berlin, Berlin, 2005, S.193 f.).

31. H. VREDELING, Bericht im Namen des Ausschusses für Fragen der Landwirtschaft über die Strukturprobleme innerhalb der Europäischen Wirtschaftsgemeinschaft einschließlich der sozialen Probleme, Europäisches Parlament, Sitzungsdokumente 1959, Luxemburg, 1959, S.1-32.

32. AdsD, EFAA000002, Deuxième Conférence des Fédérations des travailleurs agricoles (I.L.F) des Etats membres des Communautés européennes, Rome (Italie) les 25 et 26 mai 1960. Rapport du Secrétariat Syndical, S.7.

33. Vgl. J. MEYNAUD, D. SIDJANSKI, op.cit. S.349-382. 
der Konsumenteninteressen; selbst in den Agrarausschüssen waren daher die Interessen der Landarbeiter in die Interessenpolitik der Arbeitnehmer in ihrer Gesamtheit (als Konsumenten) eingebunden und ausbalanciert. Dies unterschied die Agrargewerkschaften sowohl auf nationaler wie auf europäischer Ebene deutlich von den Bauernverbänden, die die partikularen Interessen der Landwirte weitgehend ohne Rücksichtnahme auf die Belange anderer gesellschaftlicher Gruppen vertreten konnten.

Die Gewerkschaftsvertreter, deren Entsendung durch die europäischen Gewerkschaftsorganisationen (des EGS, der EFA sowie der OE-CISC) koordiniert wurde, wirkten durch ihre Teilnahme an den beratenden Ausschüssen gegenüber der Kommission durch ihren Sachverstand an der Ausarbeitung der europäischen Marktordnungen und damit an der Vollendung des europäischen Binnenmarktes in diesem Bereich in häufig mühsamer und von der Öffentlichkeit gänzlich unbeachteter Kleinarbeit mit. ${ }^{34}$ Bemerkenswerterweise trugen auch die „Verlierer“ dieser neu geschaffenen europäischen Marktordnungen, deren Preise in der Regel auf einem mittleren Niveau zwischen der bisher von den Mitgliedsstaaten praktizierten Regelungen angesiedelt wurden, insbesondere also die deutsche Gewerkschaft Gartenbau, Landund Forstwirtschaft, diesen Prozess mit.

Für die EFA als besonders bedeutsam erwies sich die Mitarbeit im „Paritätischen Ausschuss für die sozialen Probleme der ländlichen Arbeitnehmer", dessen Einrichtung die Vertreter des EGS und der EFA bereits während ihrer ersten Unterredungen mit Mansholt im Jahr 1958 und erneut auf der Konferenz von Rom über die sozialen Probleme der in der Landwirtschaft Beschäftigten, gefordert hatten. Im Mai 1963 verkündete die Kommission schließlich die Einsetzung eines solchen Ausschusses im Amtsblatt der EWG; dieser nahm ein Jahr später seine Arbeit auf. ${ }^{35}$ Anders als in den übrigen Ausschüssen waren hier ausschließlich Vertreter der Arbeitgeber und -nehmer präsent. Der Einrichtung des paritätischen Ausschusses für die Landwirtschaft institutionalisierte erstmals den sozialen Dialog zwischen den Sozialpartnern auf der Ebene der $\mathrm{E}(\mathrm{W}) \mathrm{G} .{ }^{36}$

34. Mansholt beschrieb die politischen Auseinandersetzungen um die Marktordnungen, allerdings ohne die Rolle der beratenden Ausschüsse zu erwähnen, mit einigem Sarkasmus: „Die große politische Linie geht bei diesen Sitzungen zwar verloren, aber es handelt sich dabei immerhin um handfeste materielle Interessen. Vorschläge für Eier-Handelsklassen oder Mindestgewichte für Hähnchen, für Kartoffelgrößen oder Birnenqualitäten - so was kann man nicht auf die leichte Schulter nehmen. Hinter all solchen Einzelheiten steht jeweils eine ganze Bevölkerungsgruppe“. In: S. MANSHOLT, Die Krise. Europa und die Grenzen des Wachstums, Rowohlt, Reinbek bei Hamburg, 1974, S.64.

35. Amtsblatt der EWG vom 29.05.1963, S.1534-36.

36. Allerdings war unter der Ägide des EGKS-Vertrags bereits 1952 ein paritätischer Ausschuss für den Bergbau eingerichtet worden. Dass sich auch die EU-Institutionen selbst über ihre eigene Geschichte nicht immer im Klaren sind, belegt eine Informationsbroschüre der Kommission über den sektoralen sozialen Dialog aus dem Jahr 2002, in der die Gründung des paritätischen Ausschusses für die Landwirtschaft auf das Jahr 1974 verlegt wird. Vgl. Europäische Kommission (Hrsg.), Der europäische soziale Dialog auf sektoraler Ebene, Brüssel, 2003. Dem Ausschuss gehörten neben den Vertretern der EFA auf der Arbeitnehmerseite zunächst auch drei Delegierte christlicher Gewerkschaften und ein Vertreter einer liberalen Organisation an. 
Der paritätische Ausschuss bot der EFA eine Plattform dafür, die soziale Lage der Landarbeiter mit der in der COPA organisierten Arbeitgeberseite zu diskutieren und konkrete Forderungen an sie heran zu tragen. 1966 verlangten sie erstmals, eine gemeinsame Vereinbarung zur Reduzierung der überlangen Arbeitszeiten der Landarbeiter in den EWG-Staaten auszuarbeiten. Tatsächlich kam es am 6. Juni $1968 \mathrm{zu}$ einer Absprache zwischen EFA und COPA, der zufolge die maximale Wochenarbeitszeit der Arbeitnehmer und Arbeitnehmerinnen im Ackerbau auf 45 Stunden beschränkt werden sollte. Zwar besaß diese Vereinbarung entgegen den ursprünglichen Wünschen der gewerkschaftlichen Vertreter nur empfehlenden Charakter. Doch setzte sie für die regionalen und nationalen Tarifverhandlungen eine deutliche Orientierungsmarke, auf die sich die nationalen Gewerkschaften berufen konnten. Als nicht minder bedeutsam erwies sich eine von den Präsidenten der EFA und der COPA im März 1978 unterzeichnete Absprache ,zur Harmonisierung der Arbeitszeit der ständigen Landarbeiter im Ackerbau der EWG“, welche die tariflichen Arbeitszeitabsprachen der Gewerkschaften auf nationaler Ebene ebenfalls wirksam flankierte. Sie sah eine Wochenarbeitszeit von 40 Stunden und vier Wochen bezahlten Urlaub im Jahr vor und damit Konditionen, von denen die landwirtschaftlichen Arbeitnehmer der meisten EG-Staaten zu diesem Zeitpunkt noch mehr oder minder deutlich entfernt waren. Beide Vereinbarungen ließen erstmals das künftige Potential einer europäischen Tarifpolitik in ersten Umrissen aufscheinen. ${ }^{37}$

\section{Die Reform der GAP und die EFA}

Die GAP, deren Anteil an den Gesamtausgaben der EWG im Verlauf der 1970er Jahre die $90 \%$-Marke erreichte, war kein Selbstzweck. Sie sollte die Versorgung der europäischen Verbraucher mit Lebensmitteln sicherstellen, die Produktivität in der Landwirtschaft innerhalb eines Gemeinsamen Marktes fördern und die Lebensverhältnisse, insbesondere auch die Einkommen, der ländlichen an die der städtischen Bevölkerung angleichen. Diese Zielvorstellungen der GAP wurden von den Gewerkschaften generell geteilt. Sie schienen die Möglichkeit zu eröffnen, die traditionelle Zielvorstellung der nationalen Agrargewerkschaften - Industriearbeiterlöhne für Landarbeiter - nunmehr auf europäischer Ebene zu erreichen. Die EFA unterstützte daher das Anliegen der Kommission, einen einheitlichen europäischen Agrarmarkt zu schaffen und die in allen Mitgliedsstaaten existierenden nationalen Marktordnungen und Subventionssysteme zu vereinheitlichen und durch europäische Marktordnungen zu ersetzen, und sie beteiligten sich, wie oben erwähnt wurde, insbesondere in den Beratenden Ausschüssen an ihrer konkreten Ausarbeitung.

Allerdings verlangte die EFA - auch hier wiederum im Einklang mit der Kommission und insbesondere mit Sicco Mansholt als dem spiritus rector der Gemeinsamen Agrarpolitik (und dann ihrer Reform) - schon früh eine Abkehr von einer

37. Vgl. R. FATTMANN, op.cit. S.15 f. 
reinen Preispolitik in der europäischen Landwirtschaft hin zu einer Strukturpolitik im Verein mit einer abgestimmten europäischen Sozial- und Regionalpolitik im Agrarsektor. Diese Forderungen nach einer verbesserten Strukturpolitik hatte die EFA bereits auf ihrer zweiten Konferenz am 25. und 26. Mai 1960 in Rom zum Ausdruck gebracht. Insbesondere sollten, so wurde seitens der EFA im Verlauf der 1960er Jahre immer gefordert, innerhalb des Europäischen Ausrichtungs- und Garantiefonds die für die „Ausrichtung“, d.h. für die Strukturverbesserung in der Landwirtschaft bestimmten Mittel drastisch erhöht werden.

Strukturpolitik, Regionalpolitik, Sozialpolitik, diese Trias bildete in den 1960er Jahren den unbestrittenen Kernbestand der gewerkschaftlichen Forderungen, die von den Agrargewerkschaften bereits unmittelbar nach der Gründung der EWG entwickelt worden waren und die sie in den 1960er Jahren immer wieder vortrugen und die sie in der „Entschließung der Dritten Konferenz der Landarbeitergewerkschaften (IBFG) in der EWG am 18. und 19. September in Bari“"38 noch einmal konsistent zusammengefasst hatten. Ihnen zu Folge sollte die Strukturpolitik die Landarbeiter analog zu den Beschäftigten in der Industrie - in die Lage versetzen, ,in modern eingerichteten und günstig zusammengelegten Betrieben unter sozialen Bedingungen vollbeschäftigt zu werden". Ziel der Strukturpolitik seien landwirtschaftliche Betriebe von ,ausreichender Größe“. Begrüßt wurden die Vorschläge der Kommission, die Ertragslage der landwirtschaftlichen Betriebe durch die Einrichtung von Genossenschaften zum Einkauf und Absatz und zur gemeinsamen Nutzung von Maschinen zu verbessern. Im Rahmen regionalpolitischer Pläne sollten durch eine Förderung des Wohnungsbaus auf dem Land, durch Beihilfen zur Berufsausbildung und durch eine Modernisierung der landwirtschaftlichen Haushalte die Lebensbedingungen der landwirtschaftlichen Lohnarbeitskräfte verbessert werden. Insgesamt sei auch die Sozialpolitik im Agrarbereich eine gemeinschaftliche Aufgabe, deren Angleichung überdies einen Beitrag zu fairen innergemeinschaftlichen Wettbewerbsbedingungen darstellen würde. In diesem Zusammenhang forderten die Agrargewerkschaften nichts weniger als den „Abschluss von Kollektivverträgen, die auf der Ebene der EWG [...] Mindestnormen der Lohn- und Arbeitsbedingungen der landwirtschaftlichen Lohnarbeitskräfte festlegen [sollten]". Zugleich hatte die Arbeitsgemeinschaft der Agrargewerkschaften bereits zu diesem frühen Zeitpunkt ihre Auffassung unterstrichen, ,dass eine unvertretbare Expansion der eigenen Agrarwirtschaft der EWG nicht auf Kosten der Drittländer und insbesondere nicht auf Kosten von Entwicklungsgebieten vor sich geht" .39

38. Das Dokument wiederholte und präzisierte die strukturpolitischen Forderungen der Agrargewerkschaften, die sie bereits auf ihrer 2. Konferenz am 25. und 26. Mai 1960 in Rom zum Ausdruck gebracht hatten. Vgl. auch den Bericht des Vertreters der italienischen Landarbeitergewerkschaften, Arride Rossi, Gemeinsame Strukturpolitik, unerlässliche Vorbedingung für die landwirtschaftliche Integration Europas. Exposé für die Delegierten der Dritten Konferenz der Landarbeitergewerkschaften (IBFG) in der EWG, beide enthalten in: AdsD, EFAA000003.

39. Entschliessung der Dritten Konferenz der Landarbeitergewerkschaften (IBFG) in der EWG am 18. und 19. September 1965 in Bari. 
Die im Dezember 1968 von Mansholt in seinem „Memorandum zur Reform der Landwirtschaft in der EWG" 40 vorgestellten Pläne - die eine tief greifende Reform, Modernisierung und Umstrukturierung der Agrarwirtschaft in den EWG-Mitgliedsstaaten sowie die Angleichung der nationalen Landwirtschaften und die Reduzierung der Zahl der Beschäftigten im Agrarsektor um ungefähr die Hälfte bis 1980 zum Ziel hatten - fanden so die volle Unterstützung der EFA (während sie auf wütende, teilweise gewaltsame Proteste der europäischen Bauern stießen). Vor dem Hintergrund, dass der Mansholt-Plan nicht weniger als die - kontrollierte und durch ein Bündel sozial- und regionalpolitischer Maßnahmen abgefederte - Halbierung der Zahl der in der Landwirtschaft Beschäftigten bis 1980 vorsah und damit eine erhebliche Schrumpfung auch des Organisationspotentials der Agrargewerkschaften, erscheint dies außerordentlich bemerkenswert. Die EFA entschied sich so für das Leitbild einer effektiven Landwirtschaft mit wenigen, aber gut qualifizierten und entsprechend entlohnten Arbeitnehmern und gegen die künstliche Erhaltung überkommener Strukturen, deren Abbau die nationalen Bauernverbände infolge ihres hohen Einflusses auf die nationalen Agrarministerien noch lange mit Erfolg verhindern konnten. Inwieweit Mansholts Reformvorschläge dabei direkt von den in den 1960er Jahren von der EFA im Zusammenspiel mit dem Parlament und dem WSA erarbeiteten Positionen zu einer umfassenden Struktur- und Sozialpolitik direkt beeinflusst worden waren, lässt sich dabei nur schwer abschätzen. Fakt ist jedoch, dass sich die gewerkschaftlichen Positionen im Mansholt-Plan bis in die Einzelheiten niedergeschlagen hatten und diese die Pläne des Agrarkommissars im Gegenzug dezidiert und vorbehaltlos unterstützten. ${ }^{41}$

Dabei scheint das Bündnis zwischen den Gewerkschaften und der Kommission als Motoren Europas, sowohl im Bereich der Landwirtschaft als auch branchenübergreifend, in den frühen 1970er Jahren an Schwung verloren zu haben. Dies lag zum Einen wohl daran, dass die Interessen der Gewerkschaften der nunmehr neun Mitgliedsstaaten nach der ersten Erweiterungsrunde der Gemeinschaften anders als in den anderthalb Jahrzehnten zuvor in der Entwicklung einer konsistenten Politik innerhalb der und gegenüber den Gemeinschaften nur schwer auf einen Nenner gebracht werden konnten; zum Anderen büßte auch die Kommission spätestens nach dem Ende der Übergangspräsidentschaft Mansholts im Jahr 1972 ihre ursprüngliche Rolle als Antriebskraft im Prozess der Fortentwicklung Europas - und damit als ,natürlicher' Bündnispartner der europäischen Gewerkschaftsbewegung - für geraume Zeit ein.

40. Zu Inhalt und Entstehungsgeschichte des 'Mansholt-Plans' vgl. den Beitrag Katja Seidels in diesem Band.

41. H. SCHMALZ, Ansprache zur Eröffnung der 5. Konferenz der EWG-Arbeitsgruppe der freien Landarbeitergewerkschaften gehalten am 22. September 1969 in der Westfalenhalle zu Dortmund von Helmut Schmalz, Präsident der Arbeitsgruppe, in: IISG, Nachlass Sicco L. Mansholt (1908-1995), Beleidsactiviteiten. Memo 80, Programma 1980. Stukken betreffende het Plan Mansholt inzake voorstellen tot landbouwhervorming in de EEG. 1968-1971, S.130. 


\title{
Attacking the Sacred Cow. The Norwegian Challenge to the EC's Acquis Communautaire in the Enlargement Negotiations of 1970-72
}

\author{
Robin M. ALLERS
}

On 30 June 1970 the European Communities (EC) opened membership negotiations with Great Britain, Ireland, Denmark and Norway in Luxembourg. ${ }^{1}$ This act formally ended a decade of crises most of them caused by French president Charles de Gaulle's blockade of EC-enlargement. ${ }^{2}$ Building on the agreement reached at their summit meeting in The Hague in December 1969, the Six were able to meet the candidates with a common negotiating position that emphasised unity. As the acting Council president and the president of the Commission explained in their opening speeches, the EC would insist on the acceptance of the acquis communautaire in its entirety. Furthermore, the candidates would have to agree on the Community's political goals and the further development of communitarian policies was not to be delayed because of the enlargement process. Derogations from the acquis would not be allowed, only transitional arrangements which had to be the same for all parties. ${ }^{3}$

Speaking after his colleagues from the other candidate countries, the Norwegian Foreign Minister Svenn Stray managed to shake up the audience. As could have been expected from the application text and the White Paper, his government insisted on a special scheme for Norway's agriculture which de facto amounted to a permanent exemption of his country from the Common Agricultural Policy (CAP). Similar to his fellow applicants, Stray further demanded that Norway's voice should be heard in deliberations on a Common Fisheries Policy (CFP). Like his Danish colleague he asked that already existing agreements with Nordic non-members should be maintained. Contrary to the previous speakers, however, Stray did not mention the Community's political goals that had recently been revitalised at The Hague. ${ }^{4}$ Instead he

1. This article is based on research done for my $\mathrm{PhD}$ thesis published as R.M. ALLERS, Besondere Beziehungen - Deutschland, Norwegen und Europa in der Ära Brandt (1966-1974), Dietz-Verlag, Bonn, 2009. I am grateful for comments and suggestions from participants at the workshops on the history of the CAP in Paris (5/2009) and Maastricht (4/2010), and from participants at the 2010 conference of the Oslo International Contemporary History Network.

2. W. LOTH (ed.), Crises and Compromises: The European Project 1963-1969, Nomos, Baden-Baden, 2001; N.P. LUDLOW, The European Community and the Crises of the 1960s. Negotiating the Gaullist Challenge, Routledge, London, 2006.

3. Erklärungen bei der Eröffnung der Konferenz der Europäischen Gemeinschaften und der beitrittswilligen Staaten in Luxemburg am 30. Juni 1970, in: Europa Archiv, 15-16/1970, pp.D 351-D374, here D 351-D358. See also EG-KOMMISSION: Vierter Gesamtbericht über die Tätigkeit der Gemeinschaften 1970, Brüssel 1971, p.284.

4. J. VAN DER HARST, The 1969 Hague Summit: A new start for Europe?, in: Journal of European Integration History, 2(2003), pp.5-9; J. MITTAG, W. WESSELS, Die Gipfelkonferenzen von Den Haag (1969) und Paris (1972), Meilensteine für Entwicklungstrends der Europäischen Union?, in: 